\title{
Some Theorems about $n$-Vertex Connected Graphs
}

\author{
D. M. MESNER \& M. E. WATKINS
}

\author{
Communicated by J. W. T. Youngs
}

1. Introduction. In this note, $G$ will denote an undirected graph (finite or infinite) with no loops or multiple edges. Its vertex set will be denoted by $V(G)$, or simply by $V$. If $n$ is a positive integer, a connected graph $G$ is said to be $n$-vertex connected if $|V(G)| \geqq n+1$ and if the removal of any set of fewer than $n$ vertices does not disconnect $G$. Such graphs have been investigated by Menger [3] and characterized by $\mathrm{H}$. Whitney [5] as follows:

Theorem 1. If $|V(G)| \geqq n+1$, a necessary and sufficient condition that $G$ be $n$-vertex connected is

(1-1) Given $a, b \in V(G)$, there exist $n$ arcs from $a$ to $b$ having only $a$ and $b$ in common.

In [1] and [2], G. A. Dirac has proved that some considerably generalized formulations of (1-1) are necessary for $G$ to be $n$-vertex connected. However, not all of these are sufficient. The purpose of this note is thus to generalize (1-1) as a characterization of $n$-vertex connented graphs. It is further demonstrated that the generalizations obtained are in certain respects the best possible.

2. Notation and definitions. As a general reference for the notation and vocabulary of this note, the authors recommend O. Ore [4]. If $H$ is a subgraph of $G$, written $H \subset G$, then $V(H)$ deactes the vertex set of $H$. If $U \subset V(G)$, then $G(U)$ denotes the section subgranh of $G$ with vertex set $U$.

To say that $A=A[a, b]$ is an arc shall mean that $a$ and $b$ are its terminal vertices. A family of arcs is said to be openly disjoint if the arcs in the family are pairwise disjoint except at cornmon endpoints, if any.

If $U \subset V(G)$ and $x \in V(G)$, we say $x$ is an interior vertex of $G(U)$ if all the edges incident to $x$ lie in $G(U)$, and $x$ is an exterior vertex of $G(U)$ if no edge incident to $x$ lies in $G(U)$. Otherwise, $x$ is an attachment vertex of $G(U)$.

The following equivalent formulation of an $n$-vertex connected graph, due to Ore [4], will be more convenient to use in the sequel.

Journal of Mathematics and Mechanics, Vol. 16, No. 4 (1966). 
Definition. If $n$ is a positive integer, a graph $G$ is $n$-vertex connected if for no subset $U \subset V$ does the subgraph $G(U)$ have $\mu \geqq 1$ interior vertices, $\nu \geqq 1$ exterior vertices, and $\lambda \leqq n-1$ attachment vertices.

The vertex connectivity of a graph $G$ is the largest value of $\lambda$ for which $G$ is $\lambda$-vertex connected. It will be denoted by $\lambda_{0}$.

3. An extension of Whitney's Theorem. Let $k_{1}, \cdots, k_{m}$ be a sequence of positive integers, $m \geqq 1$, and consider the following proposition concerning a graph $G$ having at least $m+1$ vertices:

$\mathrm{W}\left(k_{1}, \cdots, k_{m}\right)$ : Given any set of $m+1$ vertices $a, c_{1}, \cdots, c_{m} \varepsilon V$, there exists an openly disjoint family of $\sum_{i=1}^{m} k_{i}$ arcs from $a$ to the vertices $c_{1}, \cdots, c_{m}$ such that $k_{i}$ arcs join $a$ with $c_{i},(i=1, \cdots, m)$.

Following from a theorem of Dirac [1], we have

Theorem 2. If $G$ is n-vertex connected, then $G$ satisfies $\mathrm{W}\left(k_{1}, \cdots, k_{m}\right)$ whenever

$$
\sum_{i=1}^{m} k_{i}=n
$$

Let $m$ and $n$ be integers, $1 \leqq m \leqq n$. If $k_{1}=n-m+1$ and $k_{2}=\cdots=$ $k_{m}=1$, then $(3-1)$ holds. The statement $\mathrm{W}\left(k_{1}, \cdots, k_{m}\right)$ in this case will be denoted by $\mathrm{Y}_{m}^{n}$.

Theorem 3. Let $n$ be a positive integer. The following $2 n$ statements about the graph $G$ (having at least $n+1$ vertices) are equivalent:

$\mathrm{X}^{n}: G$ is n-vertex connected.

$\mathbf{Y}_{m}^{n}(m=1, \cdots, n)$.

$\mathbf{Z}_{m}^{n}(m=1, \cdots, n-1)$ : Given any set of $m+2$ vertices $a, b, c_{1}, \cdots, c_{m} \varepsilon V$, there exists an openly disjoint family of $n-m$ arcs joining $a$ and $b$, each of which excludes $c_{1}, \cdots, c_{m}$.

Remark. $G$ is required to have at least $n+1$ vertices in order to avoid the possibility of some $\mathbf{Y}_{m}^{n}$ or $\mathbf{Z}_{m}^{n}$ holding vacuously while $G$ is not $n$-vertex connected. If $n=1,2$, this theorem is embodied in a formulation by Ore. (See [4], p. 87.)

Proof. $\quad \mathrm{X}^{n}$ implies $\mathrm{Y}_{m}^{n}(m=1, \cdots, n)$. Use Theorem 2.

$\mathrm{Y}_{1}^{n}$ implies $\mathrm{X}^{n}$. Clearly $\mathrm{Y}_{1}^{n}$ reduces to the condition (1-1), and the result follows from Theorem 1.

$\mathbf{Y}_{m}^{n}$ implies $\mathbf{Z}_{m-1}^{n}(m=2, \cdots, n)$. Let $m+1$ distinct vertices $a, b, c_{1}, \cdots, c_{m-1}$ be given. By assumption there exists an openly disjoint family of $n$ arcs

$$
\left\{P_{i}[a, b]: i=1, \cdots, n-m+1\right\} \cup\left\{S_{i}\left[a, c_{i}\right]: i=1, \cdots, m-1\right\} .
$$

The $\operatorname{arcs} P_{1}, \cdots, P_{n-m+1}$ satisfy $\mathbf{Z}_{m-1}^{n}$ since they all exclude $c_{1}, \cdots, c_{m-1}$.

$\mathbf{Z}_{m}^{n}$ implies $\mathbf{X}^{n}(m=1, \cdots, n-1)$. Suppose $G$ is not $n$-vertex connected. Then there exists a set $A \subset V$ such that the subgraph $G(A)$ has an interior vertex $a$, an exterior vertex $b$, and $\lambda \leqq n-1$ attachment vertices $c_{1}, \cdots, c_{\lambda}$. If $\lambda \leqq m$, 
then, contrary to $Z_{m}^{n}$, no arc $P[a, b]$ can fail to contain some attachment vertex $c_{i}$. Suppose that $\lambda>m$. By $\mathbf{Z}_{m}^{n}$, there exists an openly disjoint family of $n-m$ $\operatorname{arcs} P_{i}[a, b]$ each of which excludes $c_{1}, \cdots, c_{m}$. But since $n-m>\lambda-m$, at least two of these $\operatorname{arcs} P_{i}$ must pass through some one of the vertices $c_{m+1}, \cdots, c_{\lambda}$, and hence the given family is not openly disjoint.

4. Concerning the converse to Theorem 2. It will be shown later that the converse to Theorem 2 does not hold in general. However, if $G$ is a counterexample, then $|V(G)|$ is restricted by the upper bounds given in the next theorem.

Theorem 4. Let $n$ be a positive integer. Let $G$ satisfy $\mathrm{W}\left(k_{1}, \cdots, k_{m}\right)$ where (3-1) holds and $\beta_{1}$ is the number of terms $k_{i}$ equal to 1 . If $\lambda_{0} \leqq n-1$, then

$$
|V| \leqq n-3+2\left(m-\beta_{1}\right)
$$

and

$$
\begin{array}{ll}
|V| \leqq 2 n-3, & \text { if } n \text { is even, } \\
|V| \leqq 2 n-4, & \text { if } n \text { is odd. }
\end{array}
$$

Proof. Let the sequence of positive integers

$$
k_{1}, \cdots, k_{m}
$$

satisfying (3-1) be given. Since $\lambda_{0} \leqq n-1$, there exists a set $A \subset V$ such that $G(A)$ has $\mu \geqq 1$ interior vertices, $\nu \geqq 1$ exterior vertices, and $\lambda \leqq n-1$ attachment vertices. Thus

$$
|V|=\lambda+\mu+\nu \text {. }
$$

If $\lambda<m$, let $a$ be an interior vertex of $G(A)$, let the attachment vertices be $c_{1}, \cdots, c_{\lambda}$, and let $c_{\lambda+1}$ be an exterior vertex. Then $\mathrm{W}\left(k_{1}, \cdots, k_{m}\right)$ is clearly violated. Hence suppose

$$
\lambda \geqq m \text {. }
$$

For $j=1,2, \cdots$, let $\beta_{i}$ denote the number of terms in (4-3) which are equal to $j$. Thus

$$
\sum_{i=1}^{\infty} \beta_{i}=m
$$

and

$$
\sum_{i=1}^{\infty} j \beta_{i}=n
$$

We may arrange (4-3) so that $k_{1}=\cdots=k_{\beta_{1}}=1$. Let $a$ be an interior vertex and let $c_{1}, \cdots, c_{\beta_{1}}$ be attachment vertices. By (4-5) this is possible. Assuming $\nu \geqq m-\beta_{1}$, we may let $c_{\beta_{1}+1}, \cdots, c_{m}$ be exterior vertices. Since $\mathrm{W}\left(k_{1}, \cdots, k_{m}\right)$ holds, there exists an openly disjoint family of $n-\beta_{1}$ arcs from $a$ to these exterior vertices. Hence there must exist at least $n-\beta_{1}$ attachment vertices 
distinct from $c_{1}, \cdots, c_{\beta_{1}}$, contrary to the fact that $\lambda \leqq n-1$. We conclude that $\nu \leqq m-\beta_{1}-1$, and by symmetry, $\mu \leqq m-\beta_{1}-1$. From (4-4) we obtain $|V| \leqq(n-1)+2\left(m-\beta_{1}-1\right)$, whence follows (4-1).

From (4-6) and (4-7) we obtain $2\left(m-\beta_{1}\right)=n-\beta_{1}-\sum_{i=3}^{\infty}(j-2) \beta_{i}$, which combined with (4-1) gives

$$
|V| \leqq 2 n-3-\beta_{1}-\sum_{i=3}^{\infty}(j-2) \beta_{i} .
$$

Clearly, $|V| \leqq 2 n-3$. Moreover, if $n$ is odd, then by (4-7) at least one $\beta_{i}$ for odd $j$ must be positive, whence (4-2).

Corollary. Let the sequence $k_{1}, \cdots, k_{m}$ of positive integers be given, let $\beta_{1}$ be the number of terms equal to 1 , and assume (3-1). If either

$$
|V| \geqq 2 n-2
$$

or

$$
\beta_{1} \geqq m-2,
$$

then $\mathrm{W}\left(k_{1}, \cdots, k_{m}\right)$ is a necessary and sufficient condition that $G$ be $n$-vertex connected.

Proof. $\mathrm{W}\left(k_{1}, \cdots, k_{m}\right)$ is necessary by Theorem 2 . If $(4-9)$ holds, $\mathrm{W}\left(k_{1}, \cdots, k_{m}\right)$ is sufficient by Theorem 4 .

Assuming (4-10), we remark that $\mathrm{W}\left(k_{1}, \cdots, k_{m}\right)$ reduces to $\mathrm{Y}_{n}^{n}$ if $\beta_{1}=m$ and to $\mathrm{Y}_{n-1}^{n}$ if $\beta_{1}=m-1$. Then $G$ is $n$-vertex connected by Theorem 3 . Hence suppose that $\beta_{1}=m-2$. Theorem 4 applies and (4-1) now reduces to $|V| \leqq n+1$. If $G$ is not $n$-vertex connected, then the degree of some vertex $u \varepsilon V(G)$ must be less than $n$. But this is impossible if $G$ is to satisfy $\mathrm{W}\left(k_{1}, \cdots, k_{m}\right)$ with $\sum_{i=1}^{m} k_{i}=\mathrm{n}$.

The above corollary gives sufficient conditions on the number of vertices in $G$ and on the sequence $k_{1}, \cdots, k_{m}$ in order that $\mathrm{W}\left(k_{1}, \cdots, k_{m}\right)$ be equivalent to $n$-vertex connectedness. The next theorem shows that these conditions are also necessary, i.e., that Theorem 4 is the best possible result.

Theorem 5. Let $n$ be a positive integer. Let $k_{1}, \ldots, k_{m}$ be a sequence of positive integers, $\beta_{1}$ of whose terms are equal to 1 , such that $\sum_{i=1}^{m} k_{i}=n$ and

$$
\beta_{1} \leqq m-3 \text {. }
$$

Then there exists a graph $G$ on $|V|=n-3+2\left(m-\beta_{1}\right)$ vertices which satisfies $\mathrm{W}\left(k_{1}, \cdots, k_{m}\right)$ but has vertex connectivity $\lambda_{0} \leqq n-1$.

Proof. In the complete graph on $n-3+2\left(m-\beta_{1}\right)$ vertices, decompose the vertex set into disjoint sets $V_{1}, V_{2}, V_{3}$, where $V_{2}$ contains $n-1$ vertices and each of $V_{1}, V_{3}$ contains $m-\beta_{1}-1$ vertices. Let $G$ be the graph remaining when all edges joining points of $V_{1}$ to points of $V_{3}$ are deleted. $G-G\left(V_{2}\right)$ 
is not connected, showing that $\lambda_{0} \leqq n-1$. It remains to show that $\mathrm{W}\left(k_{1}, \cdots, k_{m}\right)$ is satisfied.

Let $a, c_{1}, \cdots, c_{m} \varepsilon V(G)$ be given. For $j=1,2,3$, let $r_{i}$ denote the number of vertices $c_{i} \varepsilon V_{i}$, and let $R_{i}=\sum_{c_{i \varepsilon V}} k_{i}$. Thus

$$
r_{1}+r_{2}+r_{3}=m
$$

and

$$
R_{1}+R_{2}+R_{3}=n .
$$

We shall now prove the existence of an openly disjoint family of $n$ arcs from $a$ to $c_{1}, \cdots, c_{m}$ satisfying $\mathrm{W}\left(k_{1}, \cdots, k_{m}\right)$. Since for any arc $A[a, b] \subset G$ there exists an arc $A^{\prime}[a, b]$ of length at most 2 and such that $V\left(A^{\prime}\right) \subset V(A)$, the arcs in this family may be chosen to consist of single edges when possible and of precisely 2 edges otherwise.

Case 1: $\quad a \varepsilon V_{1} \cup V_{3}$. By symmetry we may assume $a \varepsilon V_{1}$. Then $r_{1}+r_{2}$ of the required arcs may be chosen to consist of single edges, and the remaining arcs must be of length 2 . These remaining arcs may be constructed provided there exist three disjoint sets of vertices, namely $R_{1}-r_{1}$ vertices of $V_{1} \cup V_{2}$, $R_{2}-r_{2}$ vertices of $V_{1} \cup V_{2}$, and $R_{3}$ vertices of $V_{2}$, to be used as the intermediate vertices on the arcs of length 2 from $a$ to vertices $c_{i}$ lying in $V_{1}, V_{2}$, and $V_{3}$, respectively.

Since $r_{3} \leqq m-\beta_{1}-1$, we have by (4-12), $r_{1}+r_{2} \geqq \beta_{1}+1$. Hence, $k_{i}>1$ for at least one $c_{i} \varepsilon V_{1} \cup V_{2}$, and

$$
R_{1}+R_{2} \geqq r_{1}+r_{2}+1 \text {. }
$$

In addition to vertices $c_{i}, V_{2}$ contains $n-1-r_{2}$ vertices from which $R_{3}$ vertices may be selected, since by (4-13) and (4-14), $\left(n-1-r_{2}\right)-R_{3}=$ $R_{1}+R_{2}-r_{2}-1 \geqq r_{1} \geqq 0$. Now in addition to $a$, vertices $c_{i}$, and the $R_{3}$ vertices previously selected from $V_{2}, V_{1} \cup V_{2}$ also contains $m-\beta_{1}-3+$ $n-r_{1}-r_{2}-R_{3}$ vertices. If from these we select $\left(R_{1}-r_{1}\right)+\left(R_{2}-r_{2}\right)$ vertices, by (4-11) and (4-13) there remain $m-\beta_{1}-3 \geqq 0$ vertices.

Case 2: $\quad a \varepsilon V_{2}$. Now $r_{1}+r_{2}+r_{3}$ of the required arcs may be chosen to consist of single edges. The construction of the remaining arcs is possible provided there exist three disjoint sets of vertices, namely $R_{1}-r_{1}$ vertices of $V_{1} \cup V_{2}, R_{2}-r_{2}$ vertices of $V_{1} \cup V_{2} \cup V_{3}$, and $R_{3}-r_{3}$ vertices of $V_{2} \cup V_{3}$, to be used as the intermediate vertices on the arcs of length 2 from $a$ to vertices $c_{i}$ lying in $V_{1}, V_{2}$, and $V_{3}$, respectively.

For $j=1,3$, we may select up to $m_{1}-\beta_{1}-1-r_{j}$ vertices from $V_{i}$. We next show that if $R_{i}>m-\beta_{1}-1$, then the number $R_{j}-\left(m-\beta_{1}-1\right)$ of vertices still required does not exceed the number $n-2-r_{2}$ of vertices in $V_{2}$ other than $a$ and those $c_{i} \varepsilon V_{2}$. For $j=1$, it follows from (4-11) and (4-13) that $\left(n-2-r_{2}\right)-\left[R_{1}-\left(m-\beta_{1}-1\right)\right] \geqq R_{2}-r_{2}+R_{3} \geqq 0$. The case for $j=3$ is analogous. Moreover, the selection for $j=1,3$ can be made simultaneously, since by a similar argument, 


$$
\left(n-2-r_{2}\right)-\left[R_{1}+R_{3}-2\left(m-\beta_{1}-1\right)\right] \geqq R_{2}-r_{2}+2>0 .
$$

It can be verified that the left hand member of (4-15) represents the number of vertices of $V_{1} \cup V_{2} \cup V_{3}$ other than $a, c_{1}, \cdots, c_{m}$ and the $\left(R_{1}-r_{1}\right)+\left(R_{3}-r_{3}\right)$ intermediate vertices previously selected. Since this number exceeds $R_{2}-r_{2}$, the desired family of ares can be constructed, and the proof is complete.

The example with the fewest vertices which illustrates Theorem 5 is a graph $G$ with $|V|=9$ and $\lambda_{0}=5$, constructed from the complete graph on 9 vertices by deleting the edges of a 4-cycle. $G$ satisfies $\mathrm{W}(2,2,2) . G$ is by no means unique, for if any edges of a 5-cycle on the five vertices of degree 8 are also deleted, the remaining graph $G^{\prime}$ still satisfies $\mathrm{W}(2,2,2)$ and is 5-vertex connected.

\section{BibLIOGRAPHY}

[1] G. A. Dirac, Généralisations du théorème de Menger, C. R. Acad. Sci. Paris, 250 (1960) $4252-4253$.

[2] G. A. Dirac, Extensions of Menger's Theorem, J. London Math. Soc. 38 (1963) 148-161.

[3] K. Menger, Zur allgemeinen Kurventheorie, Fund. Math., 10 (1927) 96.

[4] O. ORE, Theory of Graphs, Providence, R. I., 1962.

[5] H. Whitner, Congruent graphs and the connectivity of graphs, Amer. J. Math., 54 (1932) 150-168.

University of North Carolina Chapel Hill, North Carolina

Date Communicated: JANUARY 22, 1966 